\title{
Clinical Impact of Local Progression in Pancreatic Cancer
}

\author{
Nicholas Cardillo, BS*; Daniel M. Seible, MD*; Katherine E. Fero, MD; Andrew R. Bruggeman, MD; \\ Reith R. Sarkar, BS; Alexa Azuara, BS; Daniel R. Simpson, MD; and James D. Murphy, MD, MS
}

\begin{abstract}
Background: The high prevalence of distant metastatic disease among patients with pancreatic cancer often draws attention away from the local pancreatic tumor. This study aimed to define the complications and hospitalizations from local versus distant disease progression among a retrospective cohort of patients with pancreatic cancer. Methods: Records of 298 cases of pancreatic cancer treated at a single institution from 2004 through 2015 were retrospectively reviewed, and cancer-related symptoms and complications requiring hospitalization were recorded. Hospitalizations related to pancreatic cancer were attributed to either local or distant progression. Cumulative incidence analyses were used to estimate the incidence of hospitalization, and multivariable Fine-Gray regression models were used to identify factors predictive of hospitalizations. Results: The 1-year cumulative incidences of hospitalization due to local versus distant disease progression were $31 \%$ and $24 \%$, respectively. Among 509 recorded hospitalizations, leading local etiologies included cholangitis (10\%), biliary obstruction (7\%), local procedure complication (7\%), and gastrointestinal bleeding (7\%). On multivariable analysis, significant predictors of hospitalization from local progression included unresectable disease (subdistribution hazard ratio [SDHR], 2.42; $P<.01)$, black race (SDHR, 3.34; $P<.01)$, younger age (SDHR, 1.02 per year; $P=.01)$, tumor in the pancreatic head (SDHR, 2.19; $P<.01)$, and larger tumor size (SDHR, 1.13 per centimeter; $P=.02$ ). Most patients who died in the hospital from pancreatic cancer $(56 \%)$ were admitted for complications of local disease progression. Conclusions: Patients with pancreatic cancer experience significant complications of local tumor progression. Although distant metastatic progression represents a hallmark of pancreatic cancer, future research should also focus on improving local therapies.
\end{abstract}

J Natl Compr Canc Netw 2018;16(6):711-717 doi: 10.6004/jncen.2018.7013

Distant metastases represent a focal point of pancreatic cancer. Half of patients diagnosed with pancreatic cancer will present with metastatic disease, and most patients who present with earlier-stage disease likely harbor undetected micrometastatic disease and will ultimately develop overt distant metastases. Aside from surgery, therapies that target the local pancreatic tumor, such as radiation therapy (RT), have largely failed to improve survival. ${ }^{1-4}$ These patterns of disease spread ap-

Department of Radiation Medicine and Applied Sciences, UC San Diego Moores Cancer Center, San Diego, California.

*These authors contributed equally.

Submitted November 7, 2017; accepted for publication January 29, 2018.

The authors have disclosed that they have no financial interests, arrangements, affiliations, or commercial interests with the manufacturers of any products discussed in this article or their competitors.

Author contributions: Study concept: Cardillo, Seible, Murphy. Data curation: Cardillo, Seible, Fero, Bruggeman, Sarkar, Azuara, Murphy. propriately lead practitioners to classify pancreatic cancer as a "distant disease problem."

Despite the importance of distant metastatic disease in pancreatic cancer, the local pancreatic tumor deserves attention. Historical data describing the natural history of pancreatic cancer attribute mortality largely to systemic insults such as organ failure, thromboembolic disease, and cachexia. ${ }^{5}$ However, more recent data implicate local tumor progression as an important cause of mortality. Iacobuzio-Donahue et $\mathrm{al}^{6}$ performed rapid

Data analysis: Seible, Fero, Bruggeman, Simpson, Murphy. Investigation: Cardillo, Seible, Fero, Murphy. Methodology: Cardillo, Seible, Fero, Bruggeman, Sarkar, Azuara, Murphy. Resources: Murphy. Software: Fero, Sarkar, Murphy. Supervision: Seible, Fero, Simpson, Murphy. Validation: Seible, Bruggeman, Murphy. Visualization: Seible, Sarkar, Azuara. Manuscript preparation: Cardillo, Seible, Fero, Murphy. Critical revision: Seible, Bruggeman, Simpson, Murphy.

Correspondence: James D. Murphy, MD, MS, Department of Radiation Medicine and Applied Sciences, UC San Diego Moores Cancer Center, 3855 Health Sciences Drive, Suite 1414, La Jolla, CA 92093-0843. Email: j2murphy@ucsd.edu 
autopsies on 76 patients who died of pancreatic cancer and found that locally destructive disease was the cause of death in nearly one-third (30\%). This autopsy study helped define the link between disease progression and mortality; however, the relationship between disease progression and the corresponding clinical implications on patient quality of life (QoL) remains less well studied.

Existing clinical research describing the patterns of disease progression often fails to report the impact on patient-specific outcomes. For example, clinical trials and other retrospective series often rely on radiographic criteria (RECIST) to define the patterns of tumor progression, which may not correlate with meaningful patient outcomes. Furthermore, clinical trials often stop tracking disease progression after the first sign of disease progression. For example, if a patient develops early asymptomatic distant disease progression, then any additional locally symptomatic disease progression that occurs later would not be captured. Our study sought to complement existing research by defining the patterns of clinically meaningful disease progression over the entire disease continuum from diagnosis to death in a retrospective cohort of consecutive patients with pancreatic cancer treated at a single institution.

\section{Methods}

\section{Data Collection}

Institutional Review Board approval for this study was obtained prior to data collection. Records of 298 consecutive patients treated for pancreatic cancer at a single academic institution from 2004 through 2015 were retrospectively reviewed. Available medical records, pathology, and radiology reports were reviewed in entirety, with a focus on defining the symptom burden that patients experience with disease progression. We collected baseline demographic information and information on tumor characteristics and staging at diagnosis. Patients were classified as having resectable, unresectable, or metastatic disease at diagnosis. The resectable group included patients who underwent definitive surgery including a pancreaticoduodenectomy, distal pancreatectomy, or total pancreatectomy. The unresectable group included patients without metastatic disease at presentation who had pancreatic tumors not amenable to resection, or had surgically resectable disease but did not have surgery due to medical comorbidity or patient preference.

Patient outcome end points collected included symptoms on initial presentation, disease-related hospitalizations, disease complications, and circumstances of death. Disease-related symptoms recorded included nausea and vomiting, gastrointestinal bleeding, cancer-related pain, biliary and gastrointestinal obstructions, thromboembolic events, and depression.

Disease-related hospitalizations were classified by physician review as secondary to either local pancreatic tumor progression or distant disease progression. Hospitalization for local progression included indications such as extrahepatic biliary obstruction/ cholangitis, gastrointestinal bleed/obstruction, and pain attributable to the primary pancreatic tumor. Hospitalization for distant progression included thromboembolic events, sepsis, and distant organ failure related to distant metastases. Certain diagnoses (ie, thromboembolic disease, sepsis) can clinically be related to either local primary tumor or distant/ systemic disease, and were sorted by careful chart review. For example, hospitalization for sepsis in the setting of biliary obstruction/cholangitis was attributed to local disease progression, whereas thromboembolism in the setting of limited/resected primary tumor but widespread metastases was attributed to distant disease. For patients with both a locally advanced primary tumor and significant distant metastatic disease burden, hospitalizations were attributed to distant disease progression. When possible, we determined the circumstances of patient death in regard to cause of death, location, and whether the patient had been enrolled into hospice.

\section{Statistical Analysis}

Competing risk analyses were used to estimate the incidence of hospitalization reported 12 months from diagnosis. In these analyses, hospitalization was the event of interest, whereas death was considered a competing event. A multivariable Fine-Gray regression model was used to identify factors predictive of hospitalization for complications due to local tumor progression, and included available demographic and clinical variables that could potentially influence the risk of hospitalization (age at diagnosis, primary tumor size and location, race, ethnicity, sex, disease stage at diagnosis, and history of primary tumor ir- 
radiation). Variables in the multivariable analyses were chosen a priori and all variables were retained in the multivariable models regardless of significance on univariable analysis; $P<.05$ was considered statistically significant. All analyses were completed using SAS 9.4 (SAS Institute Inc.).

\section{Results}

\section{Cohort Characteristics}

A total of 298 consecutive patients with pancreatic adenocarcinoma were included in this study, with patient characteristics detailed in Table 1. The median follow-up for the entire study cohort was 12.0 months. Among the total cohort, $43 \%$ underwent surgical resection of their primary pancreatic tumor, and most $(81 \%)$ received chemotherapy. A smaller number of patients received RT to the primary tumor site $(30 \%)$. These patients received RT either as a planned event $(63 \%)$ or to manage a local complication such as pain, obstruction, or gastrointestinal bleed (11\%). Fourteen patients $(5 \%)$ received RT to distant metastases for symptom palliation.

\section{Symptoms}

Most patients in this cohort experienced symptoms attributable to their pancreatic cancer (Table 2). A total of 43 patients (14\%) experienced nausea and/ or vomiting at presentation. Over the course of their disease, this incidence increased to $70 \%$, including $8 \%$ who had symptoms severe enough to require hospitalization. At presentation, most patients (57\%) experienced pain, and over the course of their disease, most $(88 \%)$ experienced cancer-related pain at some point after diagnosis. Most patients who experienced pain were treated with opioid analgesia (92\%), whereas a smaller fraction (5\%) underwent RT. A total of $17 \%$ of patients had depressive symptoms at some point throughout their disease course, and every patient who experienced depressive symptoms also experienced cancer-related pain.

\section{Hospitalizations}

Of the entire study cohort, 123 patients (41\%) were hospitalized at some point after diagnosis as a result of local disease progression, and 100 patients (34\%) were hospitalized as a result of distant disease progression. Accounting for patients who were hospitalized multiple times following diagnoses, 187 (47\%)

\begin{tabular}{|c|c|}
\hline Characteristic & $\begin{array}{c}\text { Total Cohort } \\
\text { n (\%) }\end{array}$ \\
\hline \multicolumn{2}{|l|}{ Age, y } \\
\hline $18-40$ & $5(2)$ \\
\hline $41-50$ & $21(7)$ \\
\hline $51-60$ & $80(27)$ \\
\hline $61-70$ & $84(28)$ \\
\hline $71-80$ & $85(29)$ \\
\hline$\geq 81$ & $23(8)$ \\
\hline \multicolumn{2}{|l|}{ Sex } \\
\hline Male & $153(51)$ \\
\hline Female & $145(49)$ \\
\hline \multicolumn{2}{|l|}{ Race } \\
\hline White & $259(87)$ \\
\hline Black & $10(3)$ \\
\hline Asian & $17(6)$ \\
\hline Other/Unknown & $12(4)$ \\
\hline \multicolumn{2}{|l|}{ Ethnicity } \\
\hline Non-Hispanic & $245(82)$ \\
\hline Hispanic & $40(13)$ \\
\hline Other/Unknown & $13(4)$ \\
\hline \multicolumn{2}{|l|}{ Stage at diagnosis } \\
\hline Resectable & $127(43)$ \\
\hline Unresectable & $72(24)$ \\
\hline Metastatic & $99(33)$ \\
\hline \multicolumn{2}{|l|}{ Tumor size, $\mathrm{cm}$} \\
\hline$<2$ & $29(10)$ \\
\hline$\geq 2$ & $269(90)$ \\
\hline \multicolumn{2}{|l|}{ Chemotherapy } \\
\hline No & $58(19)$ \\
\hline Yes & $240(81)$ \\
\hline \multicolumn{2}{|l|}{ Surgical resection } \\
\hline No & $171(57)$ \\
\hline Yes & $127(43)$ \\
\hline \multicolumn{2}{|l|}{ Radiation therapy ${ }^{b}$} \\
\hline No & $209(70)$ \\
\hline Yes & $89(30)$ \\
\hline \multicolumn{2}{|l|}{ Hospice enrollment } \\
\hline No & $138(46)$ \\
\hline Yes & $160(54)$ \\
\hline \multicolumn{2}{|l|}{ Deceased } \\
\hline No & $29(10)$ \\
\hline Yes & $236(79)$ \\
\hline Unknown & $33(11)$ \\
\hline \multicolumn{2}{|l|}{ Location of death } \\
\hline Hospice, NOS & $42(14)$ \\
\hline Home hospice & $98(33)$ \\
\hline Home & $8(3)$ \\
\hline Inpatient & $48(16)$ \\
\hline Inpatient hospice & $20(7)$ \\
\hline Other & $2(1)$ \\
\hline Skilled nursing facility & $3(1)$ \\
\hline Skilled nursing hospice & $3(1)$ \\
\hline Unknown & $12(4)$ \\
\hline
\end{tabular}

Abbreviation: NOS, not otherwise specified.

aEach patient may have presented with $>1$ symptom.

bTo primary tumor, for any indication. 
Cardillo et al

\begin{tabular}{|lc|}
\hline & \\
\hline Table 2. Presenting Symptoms & \\
\hline Symptoms on Initial Presentation & $\mathbf{n}(\%)$ \\
\hline $\begin{array}{l}\text { Local } \\
\text { Pain }\end{array}$ \\
\hline \begin{tabular}{lc} 
Jaundice & $170(57)$ \\
\hline Nausea/Nomiting & $111(37)$ \\
\hline $\begin{array}{l}\text { Distant/Metastatic } \\
\text { Anorexia }\end{array}$ \\
\hline Fatigue & $43(14)$ \\
\hline Fever & $15(27)$ \\
\hline
\end{tabular}
\end{tabular}

aSome patients may have presented with $\geq 1$ symptom.

total admissions resulted from complications of local progression versus 138 (34\%) from distant progression and 77 (19\%) from other etiologies (Table 3). Noncancer comorbidities led to 65 hospitalizations (13\%), and complications of definitive pancreas surgery led to 45 hospitalizations (9\%). At 12 months, the cumulative incidence of hospitalization from lo- cal disease progression was 31\%, and from distant disease progression was $24 \%$ (Figure 1A). A total of 64 hospitalizations (11\%) occurred before diagnosis and were not represented in subsequent analyses, most of which were related to biliary obstruction. The most common reason for hospitalization after diagnosis from local disease progression was cholangitis $(n=53 ; 10 \%)$ followed by biliary obstruction $(\mathrm{n}=36 ; 7 \%)$, complications of local procedures (eg, drain- or stent-related infection/occlusion; $n=35$; $7 \%)$, gastrointestinal bleed $(n=34 ; 7 \%)$, and gastrointestinal obstruction $(n=23 ; 5 \%)$ (Table 3$)$. The 1 -year cumulative incidences of cholangitis, biliary obstruction, complications of local procedures, gastrointestinal bleed, and gastrointestinal obstruction are shown in Figure 1B. The cumulative incidences of hospitalization from specific complications of distant disease progression are represented in Figure 1C.

On multivariable analysis, significant predictors of hospitalization from local progression included unresectable disease (subdistribution hazard ratio

Table 3. Principal Diagnosis Requiring Hospitalizationa

\begin{tabular}{|c|c|c|c|c|}
\hline \multirow[b]{2}{*}{ Principal Diagnosis of Hospitalization } & \multirow[b]{2}{*}{$\begin{array}{c}n(\%) \\
(N=298)\end{array}$} & \multicolumn{3}{|c|}{ Disease Status at Diagnosis } \\
\hline & & $\begin{array}{c}\text { Localized } \\
\text { Resectable (\%) } \\
(\mathrm{N}=126)\end{array}$ & $\begin{array}{c}\text { Localized } \\
\text { Unresectable (\%) } \\
(\mathrm{N}=73)\end{array}$ & $\begin{array}{c}\text { Metastatic (\%) } \\
(\mathrm{N}=99)\end{array}$ \\
\hline \multicolumn{5}{|l|}{ Local } \\
\hline Cholangitis & $41(14)$ & $11(9)$ & $20(27)$ & $10(10)$ \\
\hline Biliary obstruction & $32(11)$ & $9(7)$ & $14(19)$ & $9(9)$ \\
\hline Complication of local procedure & $25(8)$ & $10(8)$ & $11(15)$ & $4(4)$ \\
\hline Bowel obstruction & $23(8)$ & $6(5)$ & $10(14)$ & $7(7)$ \\
\hline Gastrointestinal bleed & $23(8)$ & $5(4)$ & $8(11)$ & $10(10)$ \\
\hline Pain from local disease & $16(5)$ & $6(5)$ & $3(4)$ & $7(7)$ \\
\hline Other & $27(9)$ & $11(9)$ & $8(11)$ & $8(8)$ \\
\hline \multicolumn{5}{|l|}{ Distant/Metastatic } \\
\hline Infection/Sepsis & $33(11)$ & $13(10)$ & $11(15)$ & $9(9)$ \\
\hline Distant organ failure & $27(9)$ & $11(9)$ & $6(8)$ & $10(10)$ \\
\hline Thromboembolic event & $21(7)$ & $5(4)$ & $5(7)$ & $11(11)$ \\
\hline Failure to thrive & $14(5)$ & $4(3)$ & $3(4)$ & $7(7)$ \\
\hline Chemotherapy-related nausea/vomiting/diarrhea & $9(3)$ & $2(1)$ & $5(7)$ & $2(2)$ \\
\hline Pain from distant disease & $8(3)$ & $3(2)$ & $1(1)$ & $4(4)$ \\
\hline Other & $26(9)$ & $10(8)$ & $8(11)$ & $8(8)$ \\
\hline \multicolumn{5}{|l|}{ Other } \\
\hline Active noncancer comorbidity & $43(14)$ & $25(20)$ & $10(14)$ & $8(8)$ \\
\hline Postoperative complication ${ }^{\mathrm{b}}$ & $30(10)$ & $29(23)$ & $0(0)$ & $1(1)$ \\
\hline Expedite workup & $4(1)$ & $2(2)$ & $0(0)$ & $2(2)$ \\
\hline
\end{tabular}

asome patients may have presented with $\geq 1$ symptom.

bPostoperative complication following pancreaticoduodenectomy, distal pancreatectomy, or total pancreatectomy. 

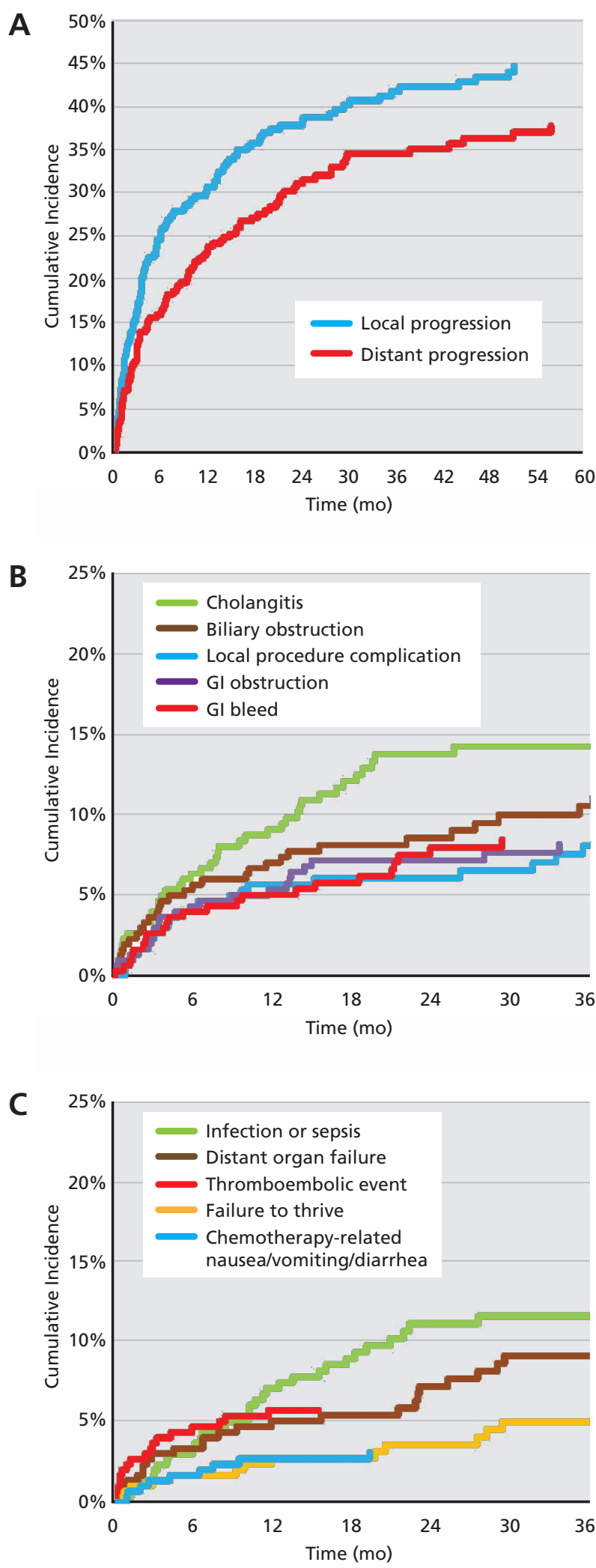

Figure 1. Cumulative incidence of hospitalizations due to (A) local versus distant progression, (B) specific comorbidities of local progression, and (C) specific comorbidities of distant progression. Abbreviation: $\mathrm{Gl}$, gastrointestinal.
[SDHR], 2.42; P<.01), black race (SDHR, 3.34; $P<.01)$, younger age (SDHR, 1.02 per year, $P=.01$ ), pancreatic head location of primary tumor (SDHR, 2.19; $P \leq .01)$, and larger tumor size (SDHR, 1.15 per centimeter; $P=.02$ ) (Table 4).

\section{Mortality}

At study end, $79 \%$ of patients were deceased $(n=236)$; median survival from diagnosis was 9.5 months. The most common setting of death was inhome hospice $(\mathrm{n}=98 ; 33 \%)$. An additional $68 \mathrm{pa}-$ tients $(23 \%)$ died while admitted or enrolled in hospice not otherwise specified, inpatient hospice, or a skilled nursing facility; 48 patients (16\%) died while hospitalized for complications related to their pancreatic cancer (Table 1). More than half of hospital inpatients $(56 \%)$ who died from disease progression were admitted primarily for complications associated with local disease progression, including gastrointestinal bleed (29\%), extrahepatic biliary obstruction $(13 \%)$, bowel obstruction (8\%), bowel ischemia $(6 \%)$, and bowel perforation (2\%).

\section{Discussion}

The key finding of this study relates to the morbidity of local disease progression in pancreatic cancer. Our study demonstrated that local disease progression resulted in higher hospitalization rates than distant disease progression. Furthermore, among the subset of patients who died as inpatients, most were admitted for complications from local disease progression. The findings of this study support those of prior research, including the autopsy study by Iacobuzio-Donahue et $\mathrm{al}^{6}{ }^{6}$ which found that locally destructive disease was the cause of death in one-third of patients. The importance of local tumor progression was further supported in a study by Nakahashi et al, ${ }^{7}$ which examined 89 patients with liver metastases and found that only a small fraction experienced hepatic failure as a result of metastatic involvement. Managing distant spread remains an important clinical goal in pancreatic cancer, although collectively these data also indicate the clinical importance of local tumor progression.

The findings in our study raise the question of whether more aggressive local therapy should be considered for treating the local pancreatic tumor. Outside of surgery, the primary locally directed ther- 
Cardillo et al

\begin{tabular}{|c|c|c|c|}
\hline Variable & SDHR & $95 \% \mathrm{Cl}$ & $P$ Value \\
\hline $\begin{array}{l}\text { Younger age at diagnosis } \\
\text { (per year) }\end{array}$ & 1.02 & $(1.01-1.03)$ & .01 \\
\hline $\begin{array}{l}\text { Larger primary tumor } \\
(\text { per } \mathrm{cm})\end{array}$ & 1.15 & $(1.02-1.24)$ & .02 \\
\hline $\begin{array}{l}\text { Pancreatic head/uncinate } \\
\text { primary }\end{array}$ & 2.19 & $(1.36-3.52)$ & $<.01$ \\
\hline \multicolumn{4}{|l|}{ Race } \\
\hline White & (Ref) & & \\
\hline Black & 3.34 & $(1.51-7.44)$ & $<.01$ \\
\hline Asian & 0.82 & $(0.36-1.84)$ & .82 \\
\hline Other & 0.57 & $(0.12-2.70)$ & .48 \\
\hline \multicolumn{4}{|l|}{ Ethnicity } \\
\hline Non-Hispanic & (Ref) & & \\
\hline Hispanic & 0.96 & $(0.67-1.68)$ & .88 \\
\hline Unknown & 2.00 & $(0.88-4.59)$ & .94 \\
\hline Male & 0.81 & $(0.56-1.16)$ & .25 \\
\hline \multicolumn{4}{|l|}{ Resectability } \\
\hline Metastatic & (Ref) & & \\
\hline Resectable & 0.68 & $(0.40-1.14)$ & .68 \\
\hline Unresectable & 2.42 & $(1.47-3.99)$ & $<.01$ \\
\hline Primary RT & 1.19 & $(0.72-1.96)$ & .82 \\
\hline
\end{tabular}

Abbreviations: RT, radiation therapy; SDHR, subdistribution hazard ratio.

apy in pancreatic cancer is RT. Unfortunately, the randomized clinical data supporting the use of RT in pancreatic cancer have shown mixed results, ${ }^{1-4}$ thus its role in the treatment of pancreatic cancer is controversial. ${ }^{8}$ The more recently reported LAP07 trial evaluated patients with locally advanced disease treated with chemotherapy followed by chemoradiation or additional chemotherapy. The results demonstrated that RT conferred a slight locoregional control benefit ( $46 \%$ vs $32 \%$ ), with no observable impact on survival. Although more aggressive RT approaches hold promise, such as stereotactic body RT $\left(\right.$ SBRT) ${ }^{9}$ or dose-escalated schemas, ${ }^{10}$ randomized evidence defining the effectiveness of these approaches is lacking. Ongoing trials will hopefully address these questions (ClinicalTrials.gov identifiers: NCT02791503, NCT01926197, NCT01821612).

Along the lines of reducing the impact of local tumor symptoms, one must consider other palliative treatments. Similar to other studies, we found that a large fraction of patients presented with biliary obstruction. ${ }^{11}$ Endoscopic stenting represents the primary approach to ameliorating biliary obstruction, although operative biliary bypass surgery also plays

a role in improving patient $\mathrm{QoL}$ and potentially survival. ${ }^{12}$ Our study also found that most patients presented with pain. Although analgesic medications represent the primary treatment, other palliative procedures may be used, such as celiac plexus neurolysis or palliative RT.

However, although these palliative treatments improve QoL, often they may not address the underlying local pancreatic tumor. Conversely, effective local therapy may not improve survival, although they could substantially improve QoL. Numerous local therapy options exist, such as conventionally fractionated RT, and more modern approaches are also available, including SBRT, ${ }^{9}$ novel intraoperative techniques such as irreversible electroporation, ${ }^{13}$ and the development of targeted radiosensitizers. ${ }^{14}$

Furthermore, understanding patients at risk of local progression represents an important goal. Iacobuzio-Donahue et $\mathrm{al}^{6}$ found that intact DPC4 genotype predicted death by local progression. ${ }^{6}$ This current study found that younger patients with larger unresectable pancreatic head tumors were most likely to experience complications of local disease progression. Clinical indicators and tumor biomarkers to help identify patients at higher risk of local tumor progression could help distinguish those who might benefit most from local therapy.

Our finding that black race independently predicts for complications of local progression deserves further consideration. The higher relative incidence of pancreatic cancer among blacks is well-documented in the literature ${ }^{15}$; however, an intrinsically more aggressive clinical course has not been widely described among this population. Furthermore, data showing shrinking outcome disparities over time support modifiable drivers of disparate disease course as playing a larger role than inherent predisposition. ${ }^{16}$ Although our study had a small proportion of black patients $(n=10)$, additional research is needed in this underserved cohort of patients.

This retrospective observational study has limitations worth considering. One of our primary end points was hospitalization from complications of pancreatic cancer disease progression. Distinguishing local from distant causes of disease progression has inherent challenges even in the prospective setting, and misclassification of this end point is possible. Additionally, the notes for patients hospitalized outside of our network may not be complete, leading 
to potential underascertainment. Our subset analysis of cause of death only focused on a small fraction of patients. We found that local disease was a major cause of death for patients who died in an inpatient setting; however, it remains entirely possible that those who died at home had different patterns of disease progression. Finally, our cohort may not represent the standard population of patients with pancreatic cancer, which raises questions surrounding generalizability. For example, UC San Diego Moores Cancer Center cares for a higher fraction of patients with resectable disease (43\%) than the general population with pancreatic cancer, due to the institution's status as a regional referral center for pancreaticobiliary surgery. Although this could potentially bias our results, our subset and multivariable analysis helped separate the impact of disease stage.

\section{Conclusions}

Despite the importance of metastatic disease, local disease progression in pancreatic cancer places a great burden on our patients. Improved combination systemic therapy regimens may work similarly on distant and local disease; however, many patients cannot tolerate these aggressive cytotoxic therapies. Although local therapies may not substantially influence survival, it is possible that an effective local treatment could substantially improve patient QoL. Thus, future research should focus on improving local therapies to help optimize patient outcomes.

\section{References}

1. Klaassen DJ, Macintyre JM, Catton GE, et al. Treatment of locally unresectable cancer of the stomach and pancreas: a randomized comparison of 5-fluorouracil alone with radiation plus concurrent and maintenance 5-fluorouracil—an Eastern Cooperative Oncology Group study. J Clin Oncol 1985;3:373-378.

2. Gastrointestinal Tumor Study Group. Treatment of locally unresectable carcinoma of the pancreas: comparison of combined-modality therapy (chemotherapy plus radiotherapy) to chemotherapy alone. J Natl Cancer Inst 1988;80:751-755.

3. Hazel JJ, Thirlwell MP, Huggins M, et al. Multi-drug chemotherapy with and without radiation for carcinoma of the stomach and pancreas: a prospective randomized trial. J Can Assoc Radiol 1981;32:164-165.

4. Chauffert B, Mornex F, Bonnetain F, et al. Phase III trial comparing intensive induction chemoradiotherapy (60 Gy, infusional 5-FU and intermittent cisplatin) followed by maintenance gemcitabine with gemcitabine alone for locally advanced unresectable pancreatic cancer. Definitive results of the 2000-01 FFCD/SFRO study. Ann Oncol 2008;19:1592-1599.

5. Mao C, Domenico DR, Kim K, et al. Observations on the developmental patterns and the consequences of pancreatic exocrine adenocarcinoma. Findings of 154 autopsies. Arch Surg 1995;130:125-134.

6. Iacobuzio-Donahue CA, Fu B, Yachida S, et al. DPC4 gene status of the primary carcinoma correlates with patterns of failure in patients with pancreatic cancer. J Clin Oncol 2009;27:1806-1813.

7. Nakahashi C, Oda T, Kinoshita T, et al. The impact of liver metastasis on mortality in patients initially diagnosed with locally advanced or resectable pancreatic cancer. Int J Gastrointest Cancer 2003;33:155-164.
8. Faris JE, Wo JY. The controversial role of chemoradiation for patients with locally advanced pancreatic cancer. Oncologist 2013;18:981-985.

9. Rosati LM, Kumar R, Herman JM. Integration of stereotactic body radiation therapy into the multidisciplinary management of pancreatic cancer. Semin Radiat Oncol 2017;27:256-267.

10. Krishnan S, Chadha AS, Suh Y, et al. Focal radiation therapy dose escalation improves overall survival in locally advanced pancreatic cancer patients receiving induction chemotherapy and consolidative chemoradiation. Int J Radiat Oncol Biol Phys 2016;94:755-765.

11. Lillemoe KD, Pitt HA. Palliation. Surgical and otherwise. Cancer 1996;78(3 Suppl):605-614

12. Sarr MG, Cameron JL. Surgical management of unresectable carcinoma of the pancreas. Surgery 1982;91:123-133.

13. Martin RC, McFarland $K$, Ellis $S$, Velanovich V. Irreversible electroporation therapy in the management of locally advanced pancreatic adenocarcinoma. J Am Coll Surg 2012;215:361-369.

14. Walker AJ, Alcorn S, Narang A, et al. Radiosensitizers in pancreatic cancer-preclinical and clinical exploits with molecularly targeted agents. Curr Probl Cancer 2013;37:301-312.

15. DeSantis CE, Siegel RL, Sauer AG, et al. Cancer statistics for African Americans, 2016: progress and opportunities in reducing racial disparities. CA Cancer J Clin 2016;66:290-308.

16. Sun $\mathrm{H}, \mathrm{Ma} H$, Hong $G$, et al. Survival improvement in patients with pancreatic cancer by decade: a period analysis of the SEER database, 19812010. Sci Rep 2014;4:6747. 